\title{
Validation of the efficacy of the NUTRISCORE for the nutritional screening of cancer patients in China
}

\author{
Junren Kang, Hailong Li, Xiaodong Shi, Enling Ma and Wei Chen * (i)
}

\begin{abstract}
Background: Malnutrition is common in cancer patients. The NUTRISCORE is a newly developed cancer-specific nutritional screening tool and was validated by comparison with the Patient-Generated Subjective Global Assessment (PG-SGA) and Malnutrition Screening Tool (MST) in Spain. We aimed to evaluate the performance of the NUTRISCORE, MST, and PG-SGA in estimating the risk of malnutrition in Chinese cancer patients.

Methods: Data from an open parallel and multicenter cross-sectional study in 29 clinical teaching hospitals in 14 Chinese cities were used. Cancer patients were assessed for malnutrition using the PG-SGA, NUTRISCORE, and MST. The sensitivity, specificity, and areas under the receiver operating characteristic curve were estimated for the NUTRISCORE and MST using the PG-SGA as a reference.

Results: A total of 1000 cancer patients were included. The mean age was 55.9 (19 to 92 years), and 47.5\% were male. Of these patients, 450 (45.0\%) had PG-SGA B and C, 29 (2.9\%) had a NUTRISCORE $\geq 5$, and 367 (36.7\%) had an MST $\geq 2$. Using the PG-SGA as a reference, the sensitivity, specificity, and area under the curve values of the NUTRISCORE were found to be $6.2,99.8 \%$, and 0.53 , respectively. The sensitivity, specificity, and area under the curve values of the MST were 50.9, 74.9\%, and 0.63, respectively. The kappa index between the NUTRISCORE and PG-SGA was 0.066, and that between the MST and PG-SGA was $0.262(P<0.05)$.
\end{abstract}

Conclusions: The NUTRISCORE had an extremely low sensitivity in cancer patients in China compared with the MST when the PG-SGA was used as a reference.

Keywords: Cancer patients, Malnutrition, Patient-generated subjective global assessment (PG-SGA), NUTRISCORE, Malnutrition screening tool (MST)

\section{Background}

Malnutrition is common in cancer patients [1,2]. Cancer and its related inflammatory factors could cause anorexia and skeletal muscle depletion. In addition, anticancer therapy may cause impaired intake, weight loss, and malnutrition. Many cancer patients could die from cancer cachexia and malnutrition [3, 4]. Early nutritional

\footnotetext{
*Correspondence: chenw@pumch.cn
}

Department of Clinical Nutrition, Peking Union Medical College Hospital, Chinese Academy of Medical Sciences and Peking Union Medical College, \#1 Shuai Fu Yuan, Beijing 100730, Dongcheng District, China diagnosis and treatment can help to intervene or treat tumor-related malnutrition, increase the tolerance of antitumor treatment, control the side effects of antitumor treatment, and improve the quality of life [5-8].

The Patient-Generated Subjective Global Assessment (PG-SGA) is a standard nutritional assessment tool for cancer patients [9-12]. However, several factors, such as cancer type, stage, and anticancer therapy, may cause malnutrition and were not considered in the PG-SGA [13]. Recently, a new nutritional screening tool called the NUTRISCORE was developed specifically 
for cancer patients and validated by reference to the PG-SGA and Malnutrition Screening Tool (MST) [14]. The NUTRISCORE was a cancer-specific malnutrition assessment tool, while PG-SGA and MST were not design for cancer patients only and they were widely used for cancer patients in clinic practice.

In the multicenter, cross-sectional study conducted in Spain, the NUTRISCORE was found to have a better performance than the MST. The NUTRISCORE had good agreement with PG-SGA (kappa index $=0.88$ ), and less time was needed for screening with the NUTRISCORE than with the PG-SGA [14]. As a fast and cancer-specific nutritional screening tool, the NUTRISCORE has also been validated in another study in Spain [15]. However, whether it can be used to predict the malnutrition risk of cancer patients in China is unclear. Therefore, we performed a multicenter, cross-sectional study to validate the performance of the NUTRISCORE and MST compared with the PG-SGA in estimating the risk of malnutrition in cancer patients in China.

\section{Methods Study design}

Data from an open parallel and multicenter crosssectional study were retrospectively analysed. Cancer patients from thoracic surgery, gastroenterology, and oncology departments were enrolled in this open parallel and multicenter cross-sectional study in 29 clinical teaching hospitals in 14 Chinese cities in 2018 [16]. The study was approved by the Ethics Committee of Peking Union Medical College Hospital (approval No. S-K 013), and all participants provided written informed consent. Cancer patients were assessed for malnutrition using the PG-SGA, NUTRISCORE, and MST.

Inclusion criteria: 1) diagnosed with an oncologic disease, 2) age over 18 years, 3) signed informed consent; 4) willing and able to complete the questionnaires. Exclusion criteria: 1) incomplete data for calculating for the PG-SGA, NUTRISCORE, and MST.

The primary objective was to evaluate the performance of the NUTRISCORE, MST, and PG-SGA in estimating the risk of malnutrition in Chinese cancer patients. The sensitivity, specificity, positive predictive values, negative predictive values, positive likelihood ratio, negative likelihood ratio, and areas under the receiver operating characteristic (ROC) curve were estimated for the NUTRISCORE and MST using the PG-SGA as a reference.

\section{Nutritional screening tools}

In this study, the NUTRISCORE, MST, and PG-SGA were used to assess and compare the nutritional status of cancer patients and to clarify the applicability of the
NUTRISCORE in the nutritional status of Chinese cancer patients.

The NUTRISCORE was used to screen the nutritional status of cancer patients and validated in the Spanish population. It consists of four parts: involuntary weight loss in the last 3 months and poor eating in the last week due to decreased appetite, tumor location/neoplasm, and oncology treatment. Patients who scored $\geq 5$ points were classified as at risk.

PG-SGA is widely used for cancer patients, developed for hospitalized patients, and recommended by the Oncology Nutrition Dietetic Practice Group of the American Dietetic Association [9]. The PG-SGA consists of patients' self-reported section, food intake, symptoms, activities and function, weight loss and medical section, disease-related nutrition state, metabolic demand, and physical examination. The PG-SGA results were classified as well-nourished (A), moderately malnourished (B), or severely malnourished $(\mathrm{C})$. For the purpose of comparison and consistency with the NUTRISCORE study [14], PG-SGA stages B and C were also classified as a nutritional risk in this study.

The MST has been widely validated in cancer patients, although it was designed for adult acute hospital patients. The MST had only two questions: Have you lost weight recently without trying? Have you been eating poorly because of a decreased appetite? Patients who scored $\geq 2$ points were classified as at risk.

\section{Data collection}

The NUTRISCORE, MST, and PG-SGA scores of cancer patients were calculated by a trained dietician using data from an open parallel and multicenter cross-sectional study. Data were abstracted and inputted independently by two trained investigators to ensure consistency and integrity.

\section{Statistical analysis}

In the NUTRISCORE study [14], the risk for malnutrition in cancer patients was $28.2 \%$ for the MST and $22.6 \%$ for the NUTRISCORE. It was calculated that approximately 459 participants would provide $95 \%$ power to detect a significant difference of $5 \%$ (two-sided $\mathrm{a}=0.05, \beta=0.1$ ).

Measurement data were expressed as the mean \pm standard deviation, and data were counted by percentage description. To determine the accuracy of the NUTRISCORE, MST, and PG-SGA and to predict malnutrition in cancer patients, the areas under the receiver operating characteristic curve (AUC) were calculated using the PG-SGA as a reference method. The sensitivity, specificity and kappa index were also estimated. All statistical tests were two-sided, and $P$ values $<0.05$ were considered statistically significant. Statistical analysis was 
performed with SPSS software (Version 19, SPSS Inc., IBM, NY, USA).

\section{Results}

A total of 1000 cancer patients were included. The mean age was $55.9 \pm 11.8$ (range, 19 to 92 years), and $47.5 \%$ $(n=475)$ were male. The proportions of cancer patients who received a college education, secondary education, and primary school education were $21.3,57.7$, and $21.0 \%$, respectively. Furthermore, $6.1 \%$ of cancer patients had a family cancer history.

All of the pathological diagnoses of cancer patients were officially collected from the medical records. Lung cancer, breast cancer, and leukemia were the most common diseases, accounting for $34.4,19.6$, and $13.1 \%$, respectively (Table 1$)$. Twenty-nine patients $(2.9 \%)$ had a NUTRISCORE $\geq 5,367$ patients (36.7\%) had an MST $\geq 2$, and 450 patients $(45.0 \%$ ) had PG-SGA B and C (Table 1 ).

With PG-SGA as a reference method, the sensitivity, specificity, positive predictive values, negative predictive values, and AUC of the NUTRISCORE were 6.2,

Table 1 Patient characteristics

\begin{tabular}{|c|c|}
\hline Characteristics & n (\%) \\
\hline Age, $y$, mean \pm SD & $55.9+11.8$ \\
\hline Range & 19-92 \\
\hline \multicolumn{2}{|l|}{ Sex } \\
\hline Male & $475(47.5)$ \\
\hline Female & $525(52.5)$ \\
\hline \multicolumn{2}{|l|}{ Education } \\
\hline Primary school & $210(21.0)$ \\
\hline Secondary Education & $577(57.7)$ \\
\hline College education & $213(21.3)$ \\
\hline \multicolumn{2}{|l|}{ Diagnose } \\
\hline Lung cancer & $344(34.4)$ \\
\hline Breast cancer & 196 (19.6) \\
\hline Gastric cancer & $78(7.8)$ \\
\hline Liver cancer & $39(3.9)$ \\
\hline Esophageal cancer & $28(2.8)$ \\
\hline Colorectal cancer & $83(8.3)$ \\
\hline Cervical cancer & $24(2.4)$ \\
\hline Ovarian cancer & $28(2.8)$ \\
\hline Leukemia & $131(13.1)$ \\
\hline Lymphoma & $39(3.9)$ \\
\hline Others & $10(0.1)$ \\
\hline Family tumor history & $60(6.1)$ \\
\hline NUTRISCORE $\geq 5$ & $29(2.9)$ \\
\hline$M S T \geq 2$ & $367(36.7)$ \\
\hline PG-SGA B + C & $450(45.0)$ \\
\hline
\end{tabular}

BMI Body Mass Index, MST Malnutrition Screening Tool, PG-SGA PatientGenerated Subjective Global Assessment
99.8, 96.6, 56.5\%, and 0.53, respectively. The sensitivity, specificity, positive predictive values, negative predictive values, and AUC of the MST were 50.9, 74.9, 62.4, $65.1 \%$ and 0.63 , respectively (Table 2). We also compared the AUC for malnutrition in different cancer groups. With PG-SGA as a reference method, the AUC of NUTRISCORE and MST were 0.53 and 0.68 respectively for malnutrition in 344 lung cancer patients. The AUC of NUTRISCORE and MST were 0.50 and 0.58 respectively for malnutrition in 196 breast cancer patients. The sensitivity, negative predictive value, and AUC of the MST were higher than those of the NUTRISCORE, while the NUTRISCORE had higher specificity and positive predictive values (Fig. 1). The kappa index between the NUTRISCORE and PG-SGA was 0.066 , and that between the MST and PG-SGA was $0.262(P<0.05)$.

\section{Discussion}

In this study, the NUTRISCORE was first validated in cancer patients in China. Among 1000 cancer patients, $2.9 \%$ had a NUTRISCORE $\geq 5,36.7 \%$ had an MST $\geq 2$, and $45.0 \%$ had PG-SGA B and C. The NUTRISCORE had higher specificity, while the sensitivity and AUC of the MST were higher when using the PG-SGA as a reference method. The MST had a higher kappa index.

Globally, the incidence and mortality of cancer patients have significantly increased. In general, cancer patients have a hypermetabolic state, increased energy consumption, skeletal muscle depletion, and weight loss [17-19]. The oropharynx malignant tumors could decrease the ability to chew or swallow [20] and the gastrointestinal cancer could cause mechanical obstruction [21, 22]. The chemotherapy lead to weight loss, and weakness [23, 24] and radiotherapy of the head and neck could cause dental caries, stomatitis, and difficulty swallowing $[25,26]$. Therefore, malnutrition in cancer patients is common and can lead to increased complications and mortality and prolonged hospital stays [3, 4, 27].

Early diagnosis of malnutrition in cancer patients is particularly important [8]. Higher sensitivity and easyto-use nutritional screening tools for cancer patients are

Table 2 Performance comparison

\begin{tabular}{lll}
\hline & NUTRISCORE & MST \\
\hline Sensitivity \% (95\% Cl) & $6.2(4.2-8.9)$ & $50.9(46.2-55.9)$ \\
Specificity \% $(95 \% \mathrm{Cl})$ & $99.8(98.8-100)$ & $74.9(71.0-78.4)$ \\
Positive predictive value \% $(95 \% \mathrm{Cl})$ & $96.6(80.4-99.8)$ & $62.4(57.2-67.3)$ \\
Negative predictive value \% $(95 \% \mathrm{Cl})$ & $56.5(53.4-59.7)$ & $65.1(61.2-68.8)$ \\
Area under the ROC curve & $0.53(0.49-0.57)$ & $0.63(0.59-0.66)$ \\
Kappa & $0.066^{*}$ & $0.262^{*}$ \\
\hline
\end{tabular}

MST Malnutrition Screening Tool, $R O C$ receiver operating characteristic. ${ }^{*} P<0.05$ 


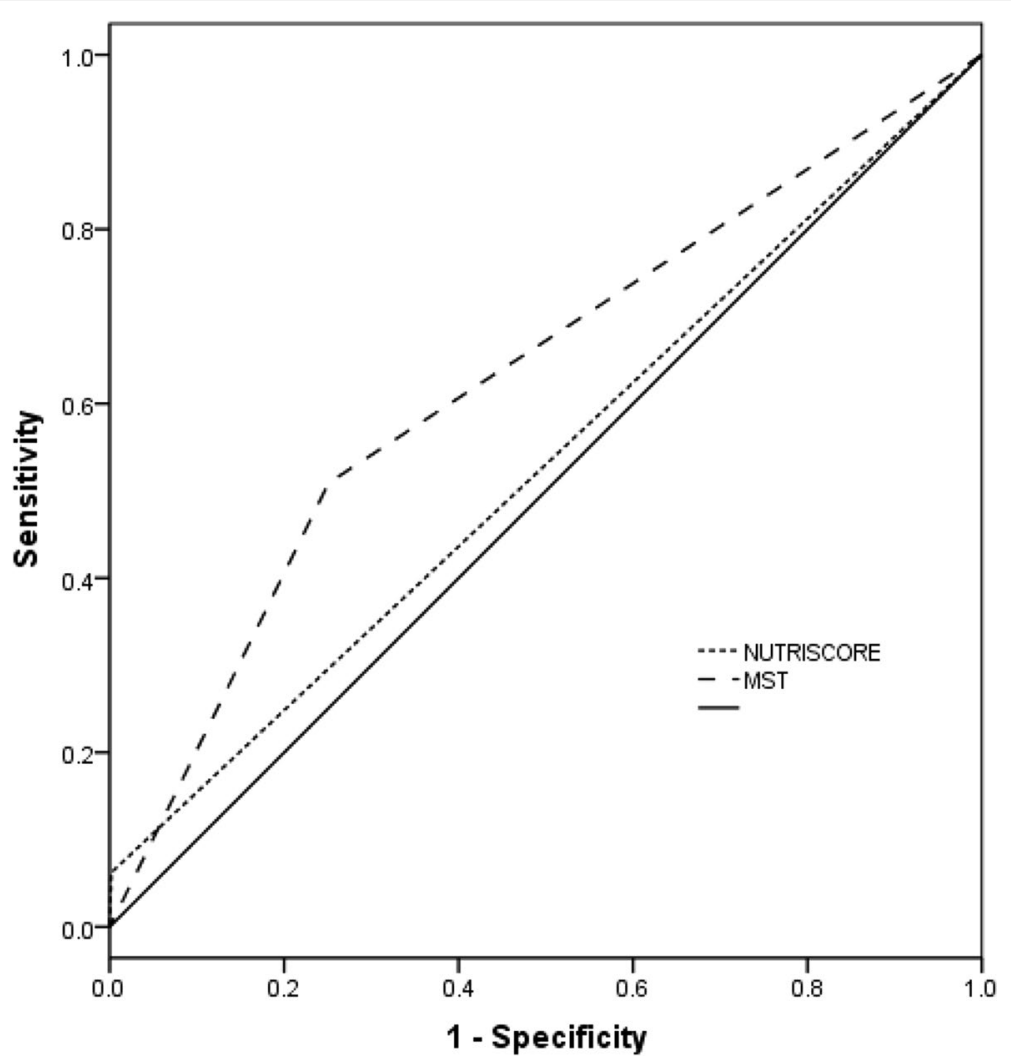

Fig. 1 Receiver operating characteristic curves of the NUTRISCORE, MST and PG-SGA

required to improve clinical outcomes. The PG-SGA is a standard nutritional assessment tool for cancer patients [28]. However, several factors, such as cancer type, stage, and anticancer therapy, may cause malnutrition and were not considered in the PG-SGA [13]. PG-SGA was not cancer-specific. In fact, the incidence of malnutrition differs across different types of cancers. The incidence of malnutrition in pancreatic cancer, gastrointestinal cancers, esophageal cancers, and hematopoietic stem cell transplantation is higher, while the incidence of malnutrition in breast cancer or prostate cancer is lower [29]. Metastatic cancers or advanced cancers were more likely to develop malnutrition [29, 30]. Anticancer chemoradiotherapy may also lead to malnutrition [31]. Therefore, cancer-specific nutritional screening tools may be needed.

As a fast and cancer-specific nutritional screening tool, the NUTRISCORE was developed and validated in the Spanish population. The NUTRISCORE not only contains weight loss and decreased appetite but also includes cancer type, location, and anticancer treatment. To validate the performance of the NUTRISCORE in cancer patients in China, we enrolled 1000 cancer patients and found that only
$2.9 \%$ of them had a NUTRISCORE $\geq 5$, while the proportion was $22.6 \%(N=394)$ in the study from Spain. In addition, the sensitivity, AUC, and kappa index of the NUTRISCORE were lower than those of the MST using the PG-SGA as a reference method, while the NUTRISCORE was found to have a better performance than the MST in the Spain study [14].

This difference may be due to the different sample distributions of the two studies. In our study, the top three cancers $(67.1 \%)$ were lung cancer $(34.4 \%)$, breast cancer (19.6\%), and leukemia (13.1\%), while the scores of breast cancer and leukemia were 0 points in the NUTRISCORE. In the NUTRISCORE study, the top three cancers (45.7\%) were abdominal and pelvic cancer (liver, biliary tract, renal and gynecologic cancer, 18.8\%), breast cancer (14.5\%) and head and neck cancer (12.4\%). Malnutrition is more common in head and neck cancer [13,29]. The distribution of the study sample may cause bias. Second, some of the patients in this study were inpatients, while the NUTRISCORE was designed for outpatient patients.

In our study, the NUTRISCORE had higher specificity and positive predictive values than the MST when using the PG-SGA as a reference method, which was consistent with a study conducted in Spain [14]. As a cancer-specific 
nutritional screening tool, the NUTRISCORE was associated with good specificity.

However, several cancer-specific factors, such as metastasis, tumor staging, and the number of courses of chemotherapy, are not included in the NUTRISCORE. Metastasis is related to malnutrition and clinical outcomes [30]. Solid tumors and hematological malignancies have different staging systems. Malnutrition related to systemic inflammation [32] was also not included in the NUTRISCORE. Whether a single cancer-specific nutritional screening tool is more specific should be discussed. For example, Onodera's prognostic nutritional index has been used for evaluating malnutrition in gastrointestinal cancer patients [33-35]. In addition, in our study, the cancer-specific NUTRISCORE did not show better performance than the MST, which only included weight loss and decreased appetite. Cancer-specific factors were also not included in the diagnostic criteria of cancer cachexia [32, 36]. In view of this, standard nutritional screening tools such as the MST and NRS2002 [37] may be suitable for cancer patients. The NRS2002 was developed for hospitalized patients and recommended by the European Society for Clinical Nutrition and Metabolism (ESPEN) [38]. It consists of three parts: severity of disease, impaired nutritional status and age. $\geq 3$ points was classified as nutritional risk [37]. The prognostic ability of the NRS2002 in cancer patients was validated in a new study published in Ann Oncol in 2021 [39]. The prognostic ability of both the NUTRISCORE and NRS2002 will be validated in our future work.

This study had some limitations. First, the distribution of cancer patients may strongly influence the results of the study. There were more breast cancer patients in this study (19.6\% vs. $14.5 \%)$, and these patients were usually not malnourished. The groups of cancer patients between the current study and NUTRISCORE study should be more comparable in theory. However, considering that the NUTRISCORE was developed as a cancer-specific nutritional screening tool for all cancer patients, not only for the cancer patients in the NUTRISCORE study, the results still hold. In addition, the study was performed with data from an open parallel and multicenter crosssectional study, which was conducted in a nonselected population of cancer patients. The distribution of cancer types might be closer to the true circumstances of cancer patients in China [40-42]. Second, most of the patients in this study were inpatients. Third, the NUTRISCORE, MST, and PG-SGA scores of cancer patients were calculated from the database, which may cause bias. Fourth, the genetic background of subjects was likely to be related to nutritional status [43]. However, the family tumor history was not discussed and excluded in the NUTRISCORE study [14] and its validated study in
Spain [15]. Family tumor history was not included in the NUTRISCORE, MST, PG-SGA or NRS2002. The genetic background of cancer subjects will be discussed in our future basic and clinical research. Further large sample studies are needed.

\section{Conclusions}

The NUTRISCORE had an extremely low sensitivity in cancer patients in China compared with the MST when using the PG-SGA as a reference. Further studies are needed.

\section{Abbreviations \\ PG-SGA: Patient-Generated Subjective Global Assessment; MST: Malnutrition Screening Tool; BMI: Body Mass Index; ROC: Receiver operating characteristic.}

\section{Acknowledgments}

Not applicable.

\section{Authors' contributions}

WC, EM and JK equally contributed to the conception and design of the research; EM and JK contributed to the design of the research; XS and $\mathrm{HL}$ contributed to the acquisition and analysis of the data; WC and JK contributed to the interpretation of the data; and JK and WC drafted the manuscript. All authors critically revised the manuscript, agree to be fully accountable for ensuring the integrity and accuracy of the work, and read and approved the final manuscript.

\section{Funding}

This study was supported by the National Natural Science Foundation of China (No. 72074222). The funding bodies played no role in the design of the study and collection, analysis, and interpretation of data and in writing the manuscript.

\section{Availability of data and materials}

The datasets used during the current study are available from the corresponding author upon reasonable request.

\section{Declarations}

\section{Ethics approval and consent to participate}

The study was approved by the Ethics Committee of Peking Union Medical College Hospital (approval No. S-K 013), and all participants provided written informed consent.

\section{Consent for publication}

Not applicable.

\section{Competing interests}

The authors state that they have no conflicts of interest.

Received: 4 February 2021 Accepted: 22 December 2021

Published online: 06 January 2022

\section{References}

1. Schneider SM, Hébuterne X. Nutritional support of the elderly cancer patient: long-term nutritional support. Nutrition. 2015;31(4):617-8.

2. Kim SH, Lee SM, Jeung HC, Lee IJ, Lee SM. The effect of nutrition intervention with oral nutritional supplements on pancreatic and bile duct cancer patients undergoing chemotherapy. Nutrients. 2019;11(5):1145. 
3. Arends J, Baracos V, Bertz H, Bozzetti F, Calder PC, Deutz N, et al. ESPEN expert group recommendations for action against cancer-related malnutrition. Clin Nutr (Edinburgh, Scotland). 2017;36(5):1187-96.

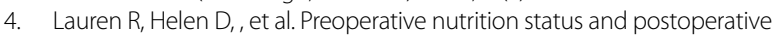
outcomes in patients undergoing cytoreductive surgery and hyperthermic intraperitoneal chemotherapy. Ann Surg Oncol. 2019;26(8):2622-2630.

5. Monika Z, Joanna KL, Leszek K, Renata Z, Sylwia M. Chemotherapy-related toxicity, nutritional status and quality of life in precachectic oncologic patients with, or without, high protein nutritional support. A prospective, randomized study. Nutrients. 2017;9(10):1108.

6. Bozzetti F. Evidence-based nutritional support of the elderly cancer patient. Nutrition (Burbank Los Angeles County, Calif). 2015;31(4):585-6.

7. Bozzetti F. Nutritional interventions in elderly gastrointestinal cancer patients: the evidence from randomized controlled trials. Support Care Cancer. 2019;27(3):721-7.

8. Arends J, Bachmann P, Baracos V, Barthelemy N, Bertz H, Bozzetti F, et al. ESPEN guidelines on nutrition in cancer patients. Clin Nutr (Edinburgh, Scotland). 2017;36(1):11-48.

9. Bauer J, Capra S, Ferguson ML. Use of the scored patient-generated subjective global assessment (pg-sga) as a nutrition assessment tool in patients with cancer. Eur J Clin Nutr. 2002;56(8):779.

10. Kim HS, Lee JY, Lim SH, Cho J, Kim SJ, Jang JH, et al. Patient-generated subjective global assessment as a prognosis tool in patients with multiple myeloma. Nutrition (Burbank, Los Angeles County, Calif). 2017;36:67-71.

11. Castillo-Martínez L, Castro-Eguiluz D, Copca-Mendoza ET, Pérez-Camargo DA, del Pilar M, Milke-García. Nutritional assessment tools for the identification of malnutrition and nutritional risk associated with cancer treatment. Rev Investig Clin. 2018;2018(70):121-5.

12. Fiol-Martínez L, Calleja-Fernández A, Pintor B, de la Maza Vidal-Casariego A, Ballesteros-Pomar MD. Comparison of two nutritional screening tools to detect nutritional risk in hematologic inpatients. Nutrition. 2017;34:97-100.

13. Wie GA, Cho YA, Kim SY, Kim SM, Bae JM, Joung H. Prevalence and risk factors of malnutrition among cancer patients according to tumor location and stage in the national cancer center in Korea. Nutrition. 2010;26(3):263-8.

14. Arribas L, Hurtós L, Sendrós MJ, Peiró I, Sánchez-Migallón JM. Nutriscore: a new nutritional screening tool for oncological outpatients. Nutrition. 2017;33:297-303.

15. Álvaro Sanz E, Garrido Siles M, Rey Fernández L, Villatoro Roldán R, Rueda Domínguez A, Abilés J. Nutritional risk and malnutrition rates at diagnosis of cancer in patients treated in outpatient settings: early intervention protocol. Nutrition (Burbank, Los Angeles County, Calif). 2019;57:148-53.

16. Li Z, Chen W, Li H, Zhao B, Chinese Oncology Nutrition Survey Group. Nutrition support in hospitalized cancer patients with malnutrition in China. Asia Pac J Clin Nutr. 2018;27(6):1216-24.

17. Gilliland TM, Villafane-Ferriol N, Shah KP, Shah RM, Tran Cao HS, Massarweh NN, et al. Nutritional and metabolic derangements in pancreatic cancer and pancreatic resection. Nutrients. 2017;9(3):243.

18. Gill AS, Kinzinger M, Bewley AF, Farwell DG, Moore MG. Malnutrition evaluation in head and neck cancer patients: practice patterns among otolaryngologists and radiation oncologists. Head Neck. 2019;41:3850-7.

19. Findlay M, Purvis M, Venman R, Luong R, Carey S. Nutritional management of patients with oesophageal cancer throughout the treatment trajectory: benchmarking against best practice. Support Care Cancer. 2020;28:5963-71.

20. Brown T, Ross $L$, Jones $L$, Hughes B, Banks M. Nutrition outcomes following implementation of validated swallowing and nutrition guidelines for patients with head and neck cancer. Support Care Cancer. 2014;22(9):2381-91.

21. Reus HDD, Hal IVD. Fully vs. partially covered selfexpandable metal stent for palliation of malignant esophageal strictures: a randomized trial (the copac study). Endoscopy. 2018;50:961-71.

22. Chen PJ, Wang L, Peng YF, Chen N, Wu AW. Surgical intervention for malignant bowel obstruction caused by gastrointestinal malignancies. World J Gastrointest Oncol. 2020;12(3):323-31.

23. Qiu Y, You J, Wang K, Cao Y, Lv Q. Effect of whole-course nutrition management on patients with esophageal cancer undergoing concurrent chemoradiotherapy: a randomized control trial. Nutrition. 2019;69:110558.

24. Kim YH, Kim GM, Son S, Song M, Lee SM. Changes in taste and food preferences in breast cancer patients receiving chemotherapy: a pilot study. Support Care Cancer. 2020;28(3):1265-75.
25. Neoh MK, Zaid ZA, Daud ZAM, Yusop NBM, Jamhuri N. Changes in nutrition impact symptoms, nutritional and functional status during head and neck cancer treatment. Nutrients. 2020;12(5):1225.

26. Hazzard E, Walton K, McMahon AT, Milosavljevic M, Tapsell L. Healthcare Professionals' perceptions of feeding tube practices for patients with head and neck cancer across 4 international radiation oncology departments. JPEN J Parenter Enteral Nutr. 2020;44(5):796-805.

27. Loan BTH, Nakahara S, Tho BA, Dang TN, Ichikawa M. Nutritional status and postoperative outcomes in patients with gastrointestinal cancer in Vietnam: a retrospective cohort study. Nutrition. 2018;48:117-21.

28. Mulasi U, Vock DM, Kuchnia AJ, Jha G, Fujioka N, Rudrapatna V. Malnutrition identified by the academy of nutrition and dietetics and american society for parenteral and enteral nutrition consensus criteria and other bedside tools is highly prevalent in a sample of individuals undergoing treatment for head and neck cancer. JPEN J Parenter Enteral Nutr. 2016;42:139-47.

29. Hébuterne X, Lemarié E, Michallet M, de Montreuil CB, Schneider SM, Goldwasser F. Prevalence of malnutrition and current use of nutrition support in patients with cancer. JPEN J Parenter Enteral Nutr. 2014;38(2):196-204.

30. Marshall KM, Loeliger J, Nolte L, Kelaart A, Kiss NK. Prevalence of malnutrition and impact on clinical outcomes in cancer services: a comparison of two time points. Clin Nutr. 2018;38:644-51.

31. Bicakli DH, Ozveren A, Uslu R, Dalak RM, Cehreli R, Uyar M, et al. The effect of chemotherapy on nutritional status and weakness in geriatric gastrointestinal system cancer patients. Nutrition. 2017;47:39-42.

32. Fearon K, Strasser F, Anker SD, Bosaeus I, Bruera E, Fainsinger RL. Definition and classification of cancer cachexia: an international consensus. Lancet Oncol. 2011:12:489-95.

33. Onodera T, Goseki N, Kosaki G. Nihon Geka Gakkai zasshi. 1984;85(9):1001-5

34. Bailón-Cuadrado M, Pérez-Saborido B, Sánchez-González J, Rodríguez-López M, Velasco-López R, Sarmentero-Prieto JC, et al. Prognostic nutritional index predicts morbidity after curative surgery for colorectal cancer. El prognostic nutritional index predice la morbilidad postoperatoria tras la cirugía curativa del cáncer colorrectal. Cirugia espanola. 2019;97(2):71-80.

35. Seretis C, Kaisari P, Wanigasooriya K, Shariff U, Youssef H. Malnutrition is associated with adverse postoperative outcome in patients undergoing elective colorectal cancer resections. J BUON. 2018;23(1):36-41.

36. Roeland EJ, Bohlke K, Baracos VE, Bruera E, Del Fabbro E, Dixon S, et al. Management of Cancer Cachexia: ASCO guideline. J Clin Oncol. 2020;38(21):2438-53.

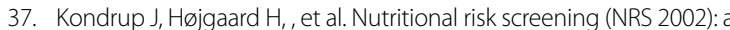
new method based on an analysis of controlled clinical trials. Clin Nutr. 2003:22:321-336

38. Kondrup J, Allison SP, Elia M, Vellas B, Plauth M, Educational and Clinical Practice Committee, European Society of Parenteral and Enteral Nutrition (ESPEN). ESPEN guidelines for nutrition screening 2002. Clin Nutr (Edinburgh, Scotland). 2003;22(4):415-21.

39. Bargetzi L, Brack C, Herrmann J, Bargetzi A, Hersberger L, Bargetzi M, et al. Nutritional support during the hospital stay reduces mortality in patients with different types of cancers: secondary analysis of a prospective randomized trial. Ann Oncol. 2021;S0923-7534(21):01993-1.

40. Guo ZQ, Yu JM, Li W, Fu ZM, Lin Y, Shi YY, et al. Survey and analysis of the nutritional status in hospitalized patients with malignant gastric tumors and its influence on the quality of life. Supp Care Cancer. 2020;28(1):373-80.

41. Song C, Cao J, Zhang F, Wang C, Guo Z, Lin Y, et al. Nutritional risk assessment by scored patient-generated subjective global assessment associated with demographic characteristics in 23,904 common malignant tumors patients. Nutr Cancer. 2019;71(1):50-60.

42. Yu JM, Yang M, Xu HX, Li W, Fu ZM, Lin Y, et al. Association between serum $C$-reactive protein concentration and nutritional status of malignant tumor patients. Nutr Cancer. 2019;71(2):240-5.

43. Vettori A, Pompucci G, Paolini B, Del Ciondolo I, Bressan S, Dundar M, et al. Genetic background, nutrition and obesity: a review. Eur Rev Med Pharmacol Sci. 2019;23(4):1751-61.

\section{Publisher's Note}

Springer Nature remains neutral with regard to jurisdictional claims in published maps and institutional affiliations. 